\title{
A near-fatal case of sepsis with an antibiotic-resistant organism complicating a routine transrectal prostate biopsy in a health care worker
}

\author{
Bryce Weber, MD; ${ }^{*}$ John Saliken, MD; ${ }^{\dagger}$ Taj Jadavji, MD; ${ }^{\ddagger}$ Robin R. Gray, MD; ${ }^{\dagger}$ Ron Moore, MD
}

\begin{abstract}
A 58-year-old physician with an elevated prostate specific antigen developed severe septic shock following a repeat transrectal prostate biopsy despite standard preoperative prophylactic protocol. This case highlights the significance of harbouring antibiotic-resistant bacteria and the risk of previous quinolone exposure. We believe this case may herald a rare but potentially serious consequence of increasingly common antibiotic resistance and that high-risk patients should be studied to determine their likelihood of carrying antibiotic-resistant flora in their genitourinary/gastrointestinal tract.
\end{abstract}

CUAJ 2008;2(5):543-5

\section{Introduction}

The spread of antibiotic resistance and the appearance of multiple antibiotic-resistant pathogenic bacteria is an increasingly prevalent problem that complicates the care of many patients. ' We present the case of a near-fatal sepsis infection in a health care worker with a history of antibiotic exposure owing to highly resistant Escherichia coli (E. coli) complicating a routine transrectal prostate biopsy despite sufficient ciprofloxacin prophylaxis. This case raises consideration that colonization by resistant organisms in high-risk patients places them at higher risk for systemic infections after usually innocuous procedures.

\section{Case presentation and management}

A 58-year-old physician with an elevated prostate-specific antigen level underwent a transrectal prostate biopsy in January 2001. Standard preoperative prophylactic protocol with ciprofloxacin $500 \mathrm{mg}$ twice daily was administered orally beginning the evening before the elective procedure and continued for 3 days. Notably, this individual spent much of his time in an oncology care unit with a large population of patients who were immunocompromised and who harboured a wide spectrum of antibiotic-resistant organisms. The patient also had 2 previous intervals of ciprofloxacin administration for prostate biopsies within the previous 2 years.

The day following the biopsy, the patient was transported to the hospital by emergency medical services after his wife found him confused, febrile and feeling generally unwell. On examination, he was systemically ill and toxic with a heart rate of 145 beats/minute, a temperature of $40^{\circ} \mathrm{C}$, an oxygen saturation of $82 \%$ and a blood pressure of 70/45 mm Hg. Physical examination revealed that the patient was diaphoretic, had a decreased level of consciousness and had ST elevation seen in the lateral leads of the electrocardiogram. Immediate resuscitation included intubation, volume and aggressive inotropic drug support, including levofed. Central venous catheterization was performed in the emergency department, and standard measures were undertaken to maintain the patient's blood pressure. Initial antibiotics in the emergency department were single doses of ampicillin, gentamicin and metronidazole. The patient was transferred to the intensive care unit (ICU) for management of severe septic shock, and multiple blood cultures were acquired. Upon transfer to the ICU, the antibiotic regimen was changed to ciprofloxacin, piperacillin-tazobactam and metronidazole. A transthoracic echocardiogram was performed, and cardiac enzymes were obtained to rule out combined septic and cardiogenic shock. Gentle diuresis was also initiated to reduce the pulmonary edema secondary to the aggressive volume resuscitation.

Blood cultures grew E. coli resistant to ampicillin, gentamicin, trimethoprimsulfamethoxazole (TMP-SMX), piperacillin, cephalothin, norfloxacin and ciprofloxacin, with intermediate sensitivity to tobramycin and amoxicillin-clavulanic acid. The organism was later found to be sensitive to meropenem and piperacillin-tazobactam. With consultation of the infectious disease service, the antibiotic regimen was changed to piperacillin-tazobactam, metronidazole and meropenem. Repeated blood cultures were acquired daily, and a CT scan of the 
Weber et al.

patient's abdomen and pelvis did not demonstrate any abscess.

As further blood cultures were available, the patient's antibiotic regimen was changed to meropenem and amikacin. With physiological support, the patient's manifestations of end-organ impairment began to resolve and he was released from the ICU after 7 days.

\section{Discussion}

Prostate biopsy is routinely performed using oral antibiotic prophylaxis with ciprofloxacin or similar drug protocol. ${ }^{2}$ There is an anticipated infection rate with this procedure of $0 \%$ to $8 \%$, with the major complication being urinary tract infections if quinolone prophylaxis is carried out to at least 4 days of total treatment. ${ }^{3-6}$ Our personal experience has shown a similar infection rate, requiring the hospitalization of $0.2 \%$ of patients. ${ }^{7}$ Yet, there have been reported cases of failure of ciprofloxacin prophylaxis in which severe or fatal sepsis has occurred owing to antibiotic-resistant bacteria. ${ }^{3,8}$ While it may be simply coincidental that a health care professional was severely affected, this patient's presentation was unique as the most acute and severe septic presentation in our experience of over 8000 transrectal ultrasound guided prostate biopsies that had been performed before this incident.

The colonization of antibiotic-resistant organisms as a result of working in an environment where such organisms are widespread is a concern for health care workers. As antibiotic resistance becomes increasingly prevalent, the extent of exposure increases. The National Nosocomial Infections Surveillance (NNIS) system reported that nosocomial E. coli isolates resistant to ciprofloxacin increased from $0.3 \%$ in 1989 to $1.4 \%$ in $1994 .{ }^{9}$ These resistant organisms are expected to be present in patients commonly subjected to antibiotics, owing to a compromised immune system creating the selective ecological pressure exerted by various antimicrobials. Specifically, new cases of fluoroquinolone resistance have been found in up to $35 \%$ of cancer patients receiving this prophylactic treatment. ${ }^{10}$ Frequently, it has been found that health care workers' hands are transiently contaminated with such organisms. ${ }^{11,12}$ If these organisms persistently colonize on health care personnel they may carry them for years, putting themselves and their contacts at risk. ${ }^{13,14}$ However, there has been little literature to investigate the possible workrelated hazard of chronic colonization of antibiotic-resistant organisms on health care workers in nonoutbreak periods. Part of the reason for this is that these studies may raise potential legal risks and work place restrictions for the participating individuals and institutions.

As a further factor of potential significance, the patient in our case study also underwent 2 prophylactic antibiotic treatments using ciprofloxacin during the previous 2 years for the same procedure. Such protocols are suspected to put selective pressure on the usual gastrointestinal and urinary flora that may raise the risk for colonization of antibiotic-resistant organisms when working in a high-exposure environment. Peña and colleagues ${ }^{15}$ reviewed a series of non-neutropenic adult patients with E. coli bacteremia and found a significant correlation between the incidence of ciprofloxacin-resistant E. coli bacteremia and previous exposure to a fluoroquinolone. In fact, the logistic regression analysis identified prior quinolone use as the only independent risk factor for ciprofloxacin-resistant $E$. coli bacteremia, with $63 \%$ of the patients having previous exposure. This is further evidence against the widespread overprescription of quinolones, which has steadily increased for the past 10 years.

This patient potentially represents a class of individuals who may have an elevated risk for colonization of antibiotic-resistant organisms and who may therefore be subject to failure of standard prophylactic antibiotic regimens before invasive procedures.

This case of rapid and nearly fatal sepsis following a common elective invasive procedure highlights the consequence of harbouring bacteria resistant to standard prophylactic regimens. We recognize that this is a rare observation, but the extremely severe sepsis combined with the unique circumstances raise a question about whether health care professionals are at higher risk of harbouring antibiotic-resistant organisms. We believe that this case may herald a rare but potentially serious consequence of increasingly common antibiotic resistance and that in some cases it may be appropriate to screen health care workers' antibiotic-resistant flora. It would also be interesting to undertake further study to determine if incidental use of antibiotics while working 
in this environment predisposes health care workers to colonization of antibiotic-resistant organisms.

While we cannot extrapolate a conclusion or recommendation for prophylactic protocol on the basis of a single incident, we do suggest that health care professionals and patients previously exposed to quinolone therapy consider additional prophylactic coverage. We believe that prospective studies are needed to assess whether other cases like this one could be prevented.

From the *Division of Urology, Department of Surgery, University of Toronto, Toronto, Ont., the †Departments of Diagnostic Imaging, Radiology and łInfectious Diseases, Foothills Hospital, University of Calgary, Calgary, Alta., and the §Department of Urology, University of Alberta, Edmonton, Alta.

This article has been peer reviewed.

Competing interests: None declared.

\section{References}

1. Haley RW, Schaberg DR, Crossley KB, et al. Extra charges and prolongation of stay altributable to nosocomial infections: a prospective interhospital comparison. Am J Med 1981;70:51-8.

2. McGinnis DE, Gomella LG. Tumors of the prostate. In: Bahnson RR, editor. Management of urologic disorders. London (UK): Mosby-Year Book Europe; 1994. p. 55.

3. Sieber PR, Rommel FM, Agusta VE, et al. Antibiotic prophylaxis in ultrasound guided transrectal prostate biopsy. J Urol 1997;157:2199-200.
4. Aus $\mathrm{G}$, Hermansson $(G$, Hugosson J, et al. Transrectal ultrasound examination of the prostate: complications and acceptance by patients. Br I Urol 1993;71:457-9.

5. Vallancien $G$, Prapotnich D, Veillon B, et al. Systematic prostatic biopsies in 100 men with no suspicion of cancer on digital rectal examination. J Urol 1991;146:1308-12.

6. Cooner WH, Mosley BR, Rutherford CL Jr, et al. Prostate cancer detection in a clinical urological practice by ultrasonography, digital rectal examination and prostate specific antigen. [discussion 1152-4.] J Urol 1990;143:1146-52.

7. Saliken JC, Gray RR, Donnelly BJ, et al. Extraprostatic biopsy improves the staging of localized prostate cancer. Can Assoc Radiol J 2000;51:114-20.

8. Gilad J, Borer A, Maimon N, et al. Failure of ciprofloxacin prophylaxis for ultrasound guided transrectal prostatic biopsy in the era of multiresistant enterobacteriaceae. J Urol 1999;161:222.

9. Wiedeman B GH, Lorian V, eds. Susceptibility to antibiotics: species incidence and trends. Baltimore: Williams \& Wilkins; 1996.

10. Carratala J, Fernandez-Sevilla A, Tubau F, et al. Emergence of fluoroquinoloneresistant Escherichia coli in fecal flora of cancer patients receiving norfloxacin prophylaxis. Antimicrob Agents Chemother 1996;40:503-5.

11. Bruun JN, Solberg CO. Hand carriage of gram-negative bacilli and Staphylococcus aureus. BMJ 1973;2:580-2.

12. Knittle MA, Eitzman DV, Baer H. Role of hand contamination of personnel in the epidemiology of gram-negative nosocomial infections. J Pediatr 1975;86:433-7.

13. Rimland D, Roberson B. Gastrointestinal carriage of methicillin-resistant Staphylococcus aureus. J Clin Microbiol 1986;24:137-8.

14. Henning KJ, Delencastre H, Eagan J, et al. Vancomycin-resistant Enterococcus faecium on a pediatric oncology ward: duration of stool shedding and incidence of clinical infection. Pediatr Infect Dis J 1996; 15:848-54.

15. Peña C, Albareda JM, Pallares R, et al. Relationship between quinolone use and emergence of ciprofloxacin-resistant Escherichia coli in bloodstream infections. Antimicrob Agents Chemother 1995;2:520-4.

Correspondence: Dr. Bryce Weber, \#102, $11660-79$ Ave., Edmonton AB T6G OP7; bweber@ualberta.ca 